\title{
Exploration on Contents and Methods of Emotionalized Design Teaching
}

\author{
Xiaohui $\mathrm{Li}^{1, \mathrm{a}}$, Cong Pang ${ }^{2, \mathrm{~b}}$, Zihan Wang ${ }^{1, \mathrm{C}}$ and Dan Huo ${ }^{1, \mathrm{~d}}$ \\ ${ }^{1}$ Dalian University of Technology, Dalian 116000, China; \\ ${ }^{2}$ Dalian University, Dalian 116000, China. \\ a lixiaohui12390@126.com, b106772196@qq.com, ' $241185484 @ q q . c o m,{ }^{d} 19647361 @ q q . c o m$
}

Keywords: Emotionalized design; teaching contents; teaching methods

\begin{abstract}
As is well-known, the information era nowadays in rapid development make the people's living conditions to be unprecedentedly complicated. The people are demanding emotional comfort more urgently with the more rapid development of science and technology, and high emotional experiences are required to be adapted with high technology. The core issue has been transferred into sensible, that is, emotionalized design from meeting the rational appeals in the design field. The concept of emotionalized design is not specifically introduced into the teaching of relevant courses of the environmental design major at the Art Department of our university, the students also often ignore this issue in their designs, as a result, the design results fall into conventional, and cannot conform to the overall trend of the current design field. This paper systematically and comprehensively illustrates the guiding role from emotionalized design principles in design process, adjusts and explores the teaching contents and methods, and really integrates emotionalized design into classrooms.
\end{abstract}

\section{Significance of Emotionalized Design Teaching Research}

\section{Contents and Roles from Emotionalized Design}

As for the social development up to now, the growing consumption demands from people and the fierce market competitions make people not just satisfied with the abundance of materials and the sharing of superficial information, but pursue deeper emotional solace from soul: satisfaction of psychological appeals, acquisition of aesthetics and enjoyment of emotional experiences, that is, pursue the emotional expressions of design. The space with a certain particular environment could be perceived and experienced, and could cause emotional reflection and feedback is called as emotionalized space, and the design undertaken on this basis is emotionalized design. [1]

The research of emotionalized design is started abroad very early. One of the most influential designers in the world, initiator of the International Cognitive Science Society and the SVP of Apple, Professor Donald Norman had started the research on design psychology and emotionalized design since the 70s of last century, his masterpiece "Emotionalized Design" takes three different design dimensions-instinct, behavior and reflection as bases, elaborates the important position and function of emotion in design, and profoundly analyzes how to integrate emotional effect into specific designs. Although the occupational features of Professor Norman enable the center of his research to focus on man-machine interaction and the field of product design on a long-term, but his theory could draw references about illustrations of the clearing-up, extracting and conclusion of emotional elements in the early stage, and could be employed in the process of environmental design to guide the subsequent in-depth design.

The instinct level, behavior level and reflection level are three dimensions of Norman's 
emotionalized design principles. The design of instinct level could be simply understood as the external forms displayed by design works, including shape, size, color and texture, etc. This level focuses on the first feeling offered to people by design in subconsciousness; the behavior level design focuses on the application fun and efficiency from the experiencer, and it could also be understood as functional needs orienting to rationality; the reflection level design focuses on the persistent influences, and it's irrelevant to the significance of the article itself, meanwhile, influenced by environment, culture, identity recognition and other factors, it's relatively complicated and changeable. This level is actually related to the long-term experiences from the experiencer. To sum up, the real emotionalized design shall be integrated in the three aforementioned levels, which is not only a standard for measuring if the design has moved the experiencer or not, but rather a criterion guiding design advancing in early stage, and it's playing an important role in further optimization to the students of art design major.[2]

\section{Current Teaching Situation of Emotionalized Design at First-Rate Universities in China}

Currently, the first-rate art design universities in China (such as Fine Arts College of Tsinghua University, etc) is focusing on the introduction of emotionalized design concept in teaching of the undergraduate specialized courses, with outstanding achievements, and deserves references.

The visual language courses opened by Fine Arts College of Tsinghua University at Grade One emphasize on expressions of personal emotion from students, the teachers are assigning propositions to students according to different stages of the courses, without any limitations on volume of materials, and are requiring the students to fully exert their own advantages in expressing the abstract feelings caused by figurative propositions. Good at using different materials and craft methods to express their own design concepts, and laying foundations for design stage of the subsequent majors. In the display design courses offered to the senior classes in the environmental art design major, the teachers are not following the conventions to require the students for traditional commercial exhibition designs, but referring to the teaching models from the interior design major of Polytechnic University of Milan, taking "joys and sorrows of life" as propositions, guiding the students for relative conceptual experimental space design, specifically introducing the principles of emotionalized design, and enabling the works from students to be more breath-taking in the emotional level.

\section{The existing problems in our environmental design teaching}

\section{The Backward Teaching Contents}

The teaching contents of our environmental art design courses are relatively backward, course task usually has no significant updates for several years, the contents of teaching presentations cannot catch up with the constantly changing trend of environmental design nowadays, therefore, students lack of interest in course contents and their enthusiasm for learning is low. The concept of emotionalized design is not explicitly introduced into the teaching contents, the design progress of students is relatively disordered and they are unable to extract, conclude and implement design concept, which cause machine-made design results and lack of personalization.

\section{The Monotonous Teaching Links}

Lively and creative teaching links can mobilize students' learning enthusiasm and achieve twice the result with half the effort. Our environmental art design teaching links are relatively monotonous, mainly consists of lectures and design practice. Without innovative and flexible 
teaching methods, lectures which depend on textbooks and presentations, are not conducive to improve the class efficiency.

\section{Too Much Emphasis on Results}

At present, the inspection way of our design courses mainly in the form of projects evaluation. Through drawing, rendering, typesetting, printing etc., students completed their works. Teachers always evaluate students only with the final visual effects but ignore the rationality of their design concept in the early stage, whether they implemented and deepened their design concepts following the principles of emotionalized design during the middle stage, whether they highlighted their design concept in the final presentations and impressed their experiencers. One-sided evaluation standard makes the students excessively focus on the final results and ignore the whole design process, which is against cultivating students' systematic design thoughts and habits.

\section{Contents and Methods of Emotionalized Design Teaching}

1. The Concept of Emotionalized Design Is Explicitly Introduced into the Teaching Contents

As mentioned above, the principles of emotionalized design are not only standards measuring if the design touches the experiencer, but rather criterions guiding design advancing in early stage, and are playing an important role in further optimization of the designs from students of the major. Therefore, the teachers shall explicitly introduce the concept of emotionalized design in routine teaching, systematically combine the theoretical framework from the predecessors to illustrate the design highlights of three levels, offer the design concept and make the students to be clear of the trend in current era satisfying the emotional appeals. And the teachers shall frequently update the contents of courseware, join in the cutting-edge design cases at home and abroad, and analyze in details one by one on representative cases according to three levels of emotionalized design principles, and deepen the impressions to students.

\section{Interaction between Teachers and Students Increased in Teaching Links}

The percentage of involvement could be properly increased in teaching links. For example, the teacher could prepare certain paper slips written with different design concepts in assignment of a certain design subject task in advance, organize the students to draw randomly, and the contents drawn will be the conceptual proposition for this student in the design. The process of drawing lots both activates the classroom atmosphere and motivates the passion from students in design. The teachers could also adopt the manner of asking the students to prepare tasks with each other, randomly set up a group by two students, and will respectively assume as Party A and Party B of the project. This manner could enable the students to exchange their roles, could cultivate the students in capacity of comprehensive reflection of issues, meanwhile, could maximally guarantee the freedom and flexibility of the design propositions, and the students could also fully exert according to their own conditions, and it will benefit the subsequent advancing of design.

\section{Methods of Emotionalized Design Teaching}

In the process of design, the students need the correct interventions and appropriate guidelines from the teachers. It is necessary to help students grasp the general direction of the design, but also to maintain its own individual design features. Teachers can follow the following process to guide students.

(1) Combined with the design concept, recall the impressive scenes of daily life resulting in mood swings. No matter if the emotional changes are strong or subtle, the real feelings and the 
authenticity of memories are very critical. According to the three dimensions of Norman's emotionalized design principles, the students could analysis the factors promoting their own mood swings objectively .

(2) Integrate the factors analyzed with spatial vocabularies.

(3) Make the research and the verification of the crowd by category. The criterions of the classification can be the factors of geographic, age, gender, occupation, etc.)

(4) Combined with the results of the survey, remove the subjective factors, find the commons and re-integrate them with spatial vocabularies.

(5) The case analysis. The research can be about the existential forms of the domestic and foreign emotionalized spaces. (Museums, exhibition halls, interactive experience space, brand stores, leisure space, public space, etc.) Focus on analyzing the level and the dimension of each space, considering about what kind of spatial vocabularies and design methods can produce the emotional fluctuations and experiences, and what kind of the effect we can achieve.

Besides the type of space,the differences of the countries, the regions,the environments and the culture also affect the application of space design language. Therefore, how to make the localization of the emotionalized design is the keypoint we need to study .

4.Assessment Methods Focus on The Implementation of Design Concepts and The Expression of Emotional Level

The assessment methods and evaluation mechanisms play a leading role in the teaching process to the students. The Assessment methods should not be limited to the drawing board in the form of print. The teachers should encourage the students to produce a complete presentation, the conceptual models and the videos which are more vivid and adapted to the individual style to start the program and guide students to pay more attention to improve the corresponding ability.

\section{Conclusion}

The emotionalized design was already not a novel word long time ago, its popularity complies with the laws of development in current era, and accords with the emotional appeals mostly thirst from people in facing the unprecedentedly complicated living conditions. As the front line in cultivating the top-end design talents, the colleges and universities shall rather integrate with the domestic and overseas design trends, shall pay attention to the teaching of emotionalized design, and actively explore the applications of emotionalized design principles in the major of environmental design.

\section{References}

[1]Norman,T.A, Emotional Design[M],Beijing, 2015(2).

[2] Norman,T.A, The Design of Everyday Things [M],Beijing, 2015(1).

[3]ZhongYang, The systematic visual presentation of the design theme - the teaching experiences with the concept board " [J]Decoration, Tsinghua University Press,2016.03,136-137 